\title{
Milliliter per Milligram per Kilogram per Day
}

National Cancer Institute

\section{Source}

National Cancer Institute. Milliliter per Milligram per Kilogram per Day. NCI Thesaurus.

Code C120819.

A unit of concentration equal to milliliter divided by milligram per kilogram per day. 